\title{
Uso del análisis de correlación canónica para la predicción de la precipitación pluvial en Centroamérica
}

\author{
Eric J. Alfaro *§ \\ *Escuela de Física, Centro de Investigaciones Geofísicas y \\ Centro de Investigaciones en Ciencias del Mar y Limnología, \\ Universidad de Costa Rica \\ §e-mail: ejalfaro@cariari.ucr.ac.cr
}

(Recibido: Enero 16 de 2007 - Aceptado: Septiembre 27 de 2007)

\section{Resumen}

Se usó un modelo basado en el análisis de correlación canónica, para explorar la capacidad de predicción de las estaciones lluviosas mayo-junio-julio (MJJ) y agosto-septiembre-octubre (ASO) en Centroamérica. Se usaron como variables predictoras las temperaturas superficiales del mar (TSM) en los océanos Atlántico y Pacífico para la región comprendida entre $112.5^{\circ} \mathrm{E}-7.5^{\circ} \mathrm{W}$ y $7.5^{\circ} \mathrm{S}-62.5^{\circ} \mathrm{N}$, de 1958 a 1998 . Para la primera parte de la estación lluviosa, MJJ, se notó que anomalías positivas (negativas) de las TSM en el Atlántico tropical están asociadas con anomalías positivas (negativas) de precipitación sobre un área amplia localizada al norte del istmo. Para la segunda parte de la estación lluviosa, ASO, signos opuestos de las anomalías en los océanos Atlántico y Pacífico, que circundan Centroamérica, estuvieron asociados con una notoria respuesta del campo de precipitación en donde anomalías positivas y negativas (negativas y positivas) de las TSM en los océanos Pacífico y Atlántico Tropical, respectivamente, tienden a estar asociadas con lóbulos de anomalías negativas (positivas) de precipitación, principalmente en la vertiente pacífica de Centroamérica. Se realizó la validación cruzada de los resultados del modelo mostrando una habilidad estadísticamente significativa sobre un área importante de la región en estudio.

Palabras clave: Correlación canónica, Predicción de precipitación, ENOS, Variabilidad del Atlántico tropical, Centroamérica.

ENVIRONMENTAL ENGINEERING

\section{Use of the canonical correlation analysis for the prediction of rainfall in Central America}

\begin{abstract}
A statistical model based on canonical correlation analysis was used to explore the predictability of early May, June, July (MJJ) and late August, September, October (ASO) rainfall seasons in Central America. Explanatory variables are seasonal Atlantic and Pacific Ocean surface sea temperature (SST) for the region inside $112.5^{\circ} \mathrm{E}-7.5^{\circ} \mathrm{W}$ and $7.5^{\circ} \mathrm{S}-62.5^{\circ} \mathrm{N}$ during $1958-1998$. For the early rainfall season, MJJ, positive (negative) tropical Atlantic SST anomalies were associated with positive (negative) rainfall anomalies over a broad area located at the north of the studied region. For the late season, ASO, opposite sign anomalies in the Atlantic and Pacific oceans that surround Central America were associated with a strong rainfall signal, in which positive \& negative (negative \& positive) SST anomalies in the Pacific \& Atlantic Tropical oceans, tend to be associated with lobes of negative (positive) rainfall anomalies mainly at Central American Pacific slope. The model results were cross validated, showing significant skill values over an important portion of the studied region.
\end{abstract}

Keywords: Canonical correlation, Rainfall prediction, ENSO, Tropical Atlantic variability, Central America. 


\section{Introducción}

Hoy en día se comprende que la variabilidad climática del campo de la precipitación en la región centroamericana está fuertemente influenciada por las variaciones de las temperaturas superficiales del mar (TSM) de los océanos circundantes al istmo (Wang, 2006; Wang et al., 2006).

Este enfoque considera de suma importancia incorporar en los estudios de variabilidad climática en Centroamérica no solamente el modo principal de variación interanual del océano Pacífico ecuatorial, es decir El Niño - Oscilación del Sur o ENOS (Waylen et al., 1994; Waylen et al., 1996a; Waylen et al., 1996b), sino también variaciones en escalas temporales interanuales y decadales de la TSM en el océano Atlántico tropical cercano a la región (Enfield \& Alfaro, 1999; Alfaro, 2000; Giannini et al., 2000; Giannini et al., 2001; Enfield et al., 2001; Waylen \& Quesada, 2001; Taylor et al., 2002; Spence et al., 2004; Poveda et al., 2006). Esto, porque el tamaño de las piscinas de aguas cálidas circundantes, con TSM mayores o iguales a los $28.5^{\circ} \mathrm{C}$ (Wang et al., 2006), y sus gradientes a través del istmo, juegan un papel muy importante en la formación de convección profunda y en la interacción de los alisios con la topografía del sistema cordillerano de Centroamérica (Enfield \& Alfaro, 1999), además de modificar algunos aspectos climáticos importantes de la circulación atmosférica suprayacente como la corriente en chorro de bajo nivel de los mares intra-americanos (Amador, 1998; Poveda \& Mesa, 1999; Wang, 2007) y la celda de Hadley (Wang, 2006).

En términos generales, las principales características del ciclo anual dominante de la precipitación en Centroamérica se pueden resumir de la siguiente manera: una marcada época de disminución de lluvias de mediados de noviembre a mediados de mayo. Luego de iniciada la estación lluviosa en mayo, se presenta un primer máximo de la misma en junio. Durante los meses de julio y agosto las precipitaciones nuevamente disminuyen, aunque en menor medida que durante noviembre-mayo. Este periodo de julio-agosto es conocido como "veranillo" o canículas (Magaña et al., 1999). Posteriormente, las precipitaciones aumentan nuevamente sobre la región, presentándose un segundo máximo en los meses de septiembre y octubre. Este segundo máximo por lo general es mayor que el de junio. El ciclo antes descrito es principalmente representativo de la vertiente del istmo sobre el oceáno Pacífico, en donde se encuentran los mayores asentamientos humanos en la región. Mayores detalles sobre la descripción de este ciclo anual pueden ser consultados en Alfaro (2002) y Taylor \& Alfaro (2005).

Un aspecto importante de considerar cuando se estudia la variabilidad climática de este ciclo anual, es el hecho de que algunos estudios sugieren que la influencia sobre el campo de precipitación del Atlántico tropical es más marcada durante el primer semestre del año, en la primavera y principios del verano boreal, influyendo directamente sobre la atmósfera suprayacente. En términos generales, para esta región oceánica, eventos cálidos (fríos) tienden a estar relacionados con un aumento (una reducción) de la precipitación sobre Centroamérica, asociado a una atmósfera más inestable (estable).

Por otro lado, la influencia del ENOS tiende a ser más significativa durante el segundo semestre del año al final del verano y comienzos del otoño boreal. $\mathrm{Su}$ influencia es principalmente en la cortante vertical del viento sobre la región, en donde en general los eventos cálidos (fríos) tienden a estar relacionados con una reducción (un aumento) de la precipitación debido a un aumento (una reducción) de la cortante vertical del viento sobre Centroamérica (Alfaro et al., 1998; Alfaro \& Cid, 1999a; Taylor et al., 2002; Spence et al., 2004).

Esta relación ya descrita entre la TSM y el campo de precipitación, además del hecho de que la memoria del sistema climático reside en el océano (ya que los cambios en la TSM tienden a suceder en una escala temporal más grande que aquellos de las variables atmosféricas), ha permitido la exploración de la predicción de la precipitación en la región. Un ejemplo de la diferencia en la respuesta a los cambios entre el océano y la atmósfera es el ENOS, el cual en algunos trabajos es explicado como un oscilador retardado debido a 
que los ajustes dinámicos en el océano son más lentos que los cambios experimentados en el campo del viento superficial en el Pacífico ecuatorial (Barry \& Carleton, 2001).

En Centroamérica y el Caribe se ha usado el campo de TSM como predictor, a través de distintos modelos estadísticos, por medio de la construcción de distintos índices oceánicos y de precipitación que capturan la mayor parte de la variabilidad encontrada tanto en el conjunto de las variables independientes (predictores) como en el de las variables dependientes (predictantes) (Alfaro \& Cid, 1999b; Alfaro \& Soley, 2001; Ashby et al., 2005).

Debido a lo anterior, el objetivo de este trabajo es el de usar un modelo basado en el análisis de correlación canónica (ACC) para explorar las asociaciones climáticas y la capacidad predictiva de las estaciones lluviosas mayo-junio-julio (MJJ) y agosto-setiembre-octubre (ASO) en Centroamérica. Estas estaciones climáticas se escogieron para capturar los dos picos del comportamiento bimodal del ciclo anual dominante de la lluvia en la región descrito anteriormente (Magaña et al., 1999, Alfaro, 2002; Taylor \& Alfaro, 2005). En este trabajo, se usaron como variables predictoras las Temperaturas superficiales del mar (TSM) en los océanos Atlántico y Pacífico. La aproximación estadística empleada en este análisis del ACC tiene dos ventajas principales. La primera es que no asume una relación estacionaria a lo largo del ciclo anual entre los campos usados y la segunda es que la variabilidad presente en ellos no es expresada a través del uso de índices, seleccionados o construidos previamente, sino más bien por medio del uso de los modos de variabilidad más correlacionados en ambos campos.

En la Sección 2 se describen con más detalle los datos y la metodología empleados y en la Sección 3 se presentan los principales resultados y su discusión. Por último, la Sección 4 contiene las conclusiones de este estudio.

\section{Datos y metodología}

El período de análisis de todas las series de tiempo mensuales empleadas en este trabajo es enero de
1958 a diciembre de 1998 (41 años).

Los datos de precipitación usados como predictantes fueron compilados por el Centro de Ciencias de la Atmósfera en la UNAM, México. Este conjunto de datos se produjo a partir de los registros de estaciones pluviométricas, datos de satélite y salidas de modelos numéricos, combinados en una rejilla con una resolución espacial de $0.5^{\circ}$ de latitud $\times 0.5^{\circ}$ de longitud. En éste estudio, se consideraron sólo aquellos puntos de la rejilla sobre o muy cercanos al istmo de Centroamérica en la región comprendida entre 78.0 - $95.0^{\circ} \mathrm{W}, 7.5-21.5^{\circ} \mathrm{N}$, para un total de 337 series de tiempo (Figura 1). Este conjunto de datos ha sido ampliamente usado en diversos estudios climáticos regionales (e.g., Magaña et al., 1999; Taylor et al., 2002; Alfaro, 2002; Ashby et al., 2005).

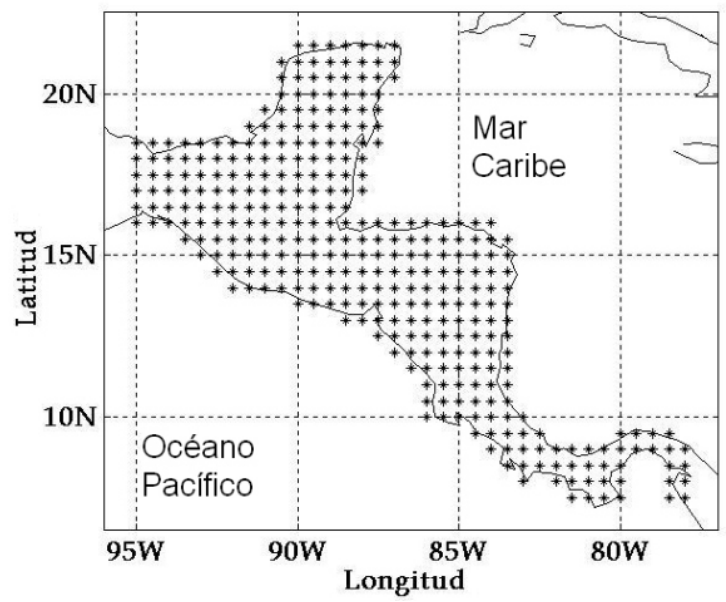

Figura 1. Los asteriscos representan la localización geográfica de los puntos de rejilla para las series de tiempo de precipitación usadas en el estudio.

Los datos usados como predictores corresponden al conjunto de datos de anomalías de TSM obtenidos del Instituto Internacional para la Investigación de la Predicción Climática (o IRI, por sus siglas en inglés), que combina los análisis hechos por Kaplan et al. (1998) y Reynolds \& Smith (1994), en una rejilla de resolución de $5^{\circ}$ de latitud $\times 5^{\circ}$ de longitud para la región comprendida entre $112.5^{\circ} \mathrm{E}-7.5^{\circ} \mathrm{W}, 7.5^{\circ} \mathrm{S}-62.5^{\circ} \mathrm{N}$ de los océanos Pacífico y Atlántico.

En este trabajo, el ACC se usó para relacionar patrones en el campo de los predictores (TSM) con patrones en el campo de las variables predichas, 
(predictandos) como la precipitación, siguiendo el procedimiento estadístico descrito por Gershunov \& Cayan (2003) y Alfaro et al. (2006), el cual se resume en los siguientes párrafos.

Con esta aproximación estadística, el ACC relaciona observaciones contemporáneas (ajustes de especificación) o anteriores (ajustes de predicción) del campo de TSM con valores observados del campo de precipitación. Las distintas estaciones climáticas del año consideradas se calcularon como el promedio de tres meses consecutivos (o sea, trimestres).

La hipótesis implícita del análisis es que patrones anómalos del campo de TSM actúan como impulsores de la circulación atmosférica de gran escala que influyen en las anomalías locales de precipitación. Estos patrones anómalos de TSM cubren un rango amplio de variabilidad temporal que abarca desde escalas interanuales hasta decadales.

El método estadístico empleado consta de tres pasos principales (ver Gershunov \& Cayan, 2003 para más detalles). Primero, los campos del predictor y del predictante son prefiltrados utilizando cada uno el mismo número de $p$ componentes principales. Segundo, los patrones de variabilidad en ambos campos, predictor y predictante, representados por sus respectivas $p$ componentes principales (CP), se relacionan entre sí por medio de $q$ correlaciones derivadas del ACC. Finalmente, se analiza un número razonable de combinaciones de $p$ y $q$ para definir el modelo estadístico óptimo, considerando la capacidad predictiva de cada modelo por medio de la técnica de validación cruzada. Los valores óptimos de $p$ y $q$ que se escogen para un modelo en particular, son aquellos que muestran en promedio la máxima capacidad predictiva en el dominio espacial analizado del predictante.

El número de modos para los modelos de especificación y de predicción se optimizó en forma separada, por lo que el número de modos para uno no es necesariamente igual que para el otro. Cabe destacar que, para un modelo en particular, la combinación de $p$ y $q$ es aproximada, ya que estos valores se seleccionan a partir de una superficie de valores de capacidad predictiva que, por lo general, no varía abruptamente, por lo que modelos con una complejidad similar al par $p-q$ escogido tendrán una capacidad predictiva promedio similar. El número de patrones $p$ y el de las relaciones seleccionadas $q$ se tomaron de un rango que varió de 2 a 17, con el fin de evitar un sobreajuste del modelo. Por último, los resultados obtenidos proveen estimados realistas de la habilidad predictiva de la precipitación, obtenidos a partir de combinaciones lineales del campo de TSM, debido a que las estimaciones de esta habilidad fueron realizadas usando la técnica de validación cruzada. La validación cruzada (ver por ejemplo, Wilks, 1995), es una técnica de remuestreo que opera en forma similar a las pruebas de bootstrap y permutación, dividiendo repetidamente el conjunto total de datos en un subconjunto de control y otro de verificación. Lo más común es que el primero sea de tamaño $n-1 \mathrm{y}$ el segundo de 1, haciendo $n$ particiones diferentes de los datos. La forma de operar de esta técnica es a través de las siguientes etapas:

Etapa (1). Se eliminan los primeros datos de la variable dependiente y el o los primeros datos de las variables independientes y se recalcula el modelo con los $n-1$ datos restantes o de control. A este modelo se le llama modelo reducido.

Etapa (2). Usando el modelo reducido calculado en la etapa (1), se estiman los valores eliminados de la variable dependiente usando los datos eliminados de las variables independientes.

Etapa (3). Se reincorporan los datos eliminados de las variables dependientes e independientes al conjunto total de datos y se eliminan los siguientes datos en las series de tiempo, repitiéndose el proceso de los puntos 1) y 2) en forma sucesiva hasta tener $n$ modelos reducidos y $n$ datos estimados, donde $n$ es la longitud de las series de tiempo. En nuestro caso, el proceso se realizó con $n=41$, correspondiente a los 41 años de análisis.

\section{Resultados y discusión}

Como un primer paso y como una forma de comparar los resultados obtenidos para MJJ y ASO con otras épocas del año, el ACC se empleó para calcular el ciclo anual de la habilidad estadística promedio en la predicción de la 


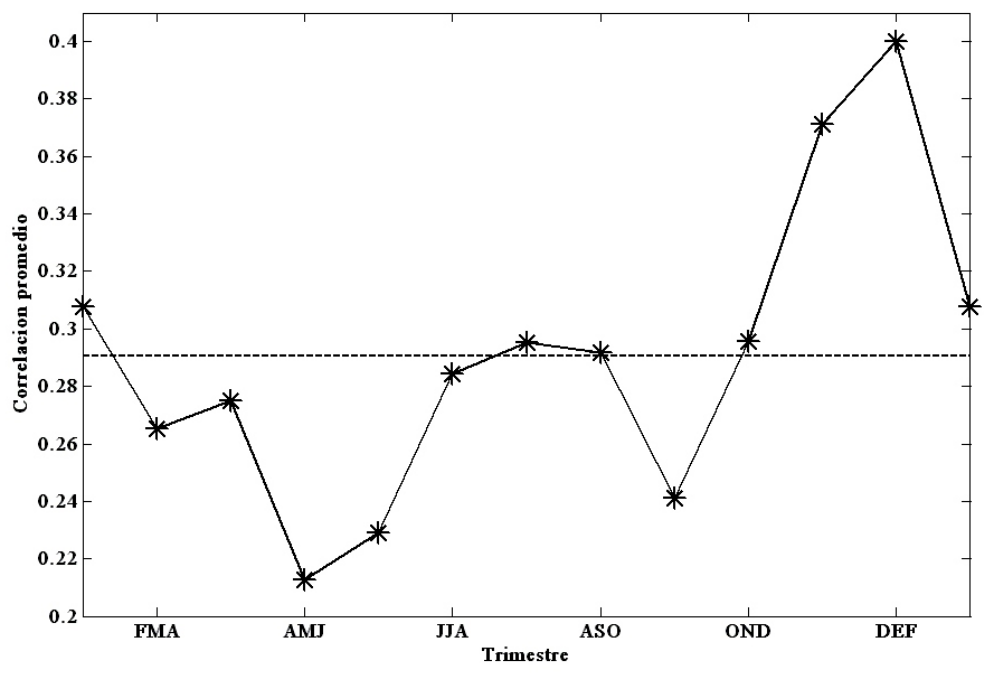

Figura 2. Ciclo anual de la habilidad estadística predictiva promedio del campo de precipitación en Centroamérica. Los trimestres usados en el ACC para el campo de los predictores (TSM) son los mismos que se consideran para el campo del predictando (precipitación), es decir: EFM, FMA, $M A M, A M J, \ldots, D E F$. Se incluyó como último valor de la derecha en esta figura nuevamente al trimestre EFM para facilitar su comparación con aquel de DEF. La línea a trazos horizontal representa el promedio de este ciclo anual (0.29).

precipitación de los modelos, usando en forma simultánea los campos de la TSM y de la precipitación para los diferentes trimestres del año (Figura 2). El valor menor fue para AMJ con 0.21 y el mayor para DEF con 0.40 , siendo el promedio de este ciclo anual de 0.29 . La alta capacidad predictiva del trimestre DEF es especialmente importante para la vertiente del Caribe en Centroamérica en donde mucha de la precipitación registrada durante este semestre es ocasionada por el paso de frentes fríos sobre la región (Alfaro, 2002). Los valores obtenidos para MJJ y ASO fueron de 0.23 y de 0.29 , respectivamente, en donde el principal mecanismo de producción de lluvias durante estos dos trimestres son sistemas mesoescalares como los de brisa marina, inmersos en una atmósfera de por sí inestable debido a la cercanía de la zona de convergencia inter-tropical al istmo (Alfaro, 2002). Vale la pena destacar que, estos valores de capacidad predictiva de la Figura 2 representan el promedio sobre toda la región del predictante y que al estudiar su patrón espacial se pueden encontrar regiones con habilidades predictivas mayores.

Posteriormente, se ajustaron los modelos predictivos para MJJ y ASO. Para la predicción de la precipitación de MJJ, se consideraron los trimestres previos de TSM desde OND hasta AMJ (junto con el trimestre simultáneo ya descrito de MJJ), mientras que, para la predicción de la precipitación de ASO, se consideraron los trimestres previos de TSM desde EFM hasta JAS (junto con el trimestre simultáneo de ASO). La habilidad estadística promedio de estos modelos se presenta en la Figura 3, en la cual se nota que la habilidad para ASO fue mayor que la encontrada para MJJ. El valor más bajo de habilidad para la predicción de ASO fue de 0.24 mientras que el más alto fue de 0.31 , usando los trimestres de AMJ y JAS como predictores, respectivamente. Por otro lado, para la predicción de MJJ, el valor más bajo de habilidad encontrado fue de 0.18 mientras que el más alto fue de 0.24 , usando los trimestres de NDE y AMJ como predictores, respectivamente. Nótese que, en general, el trimestre de ASO fue mejor predicho que aquel de MJJ para los diferentes rezagos considerados (Figura 3).

Específicamente, para la predicción de los trimestres de MJJ y ASO, la Figura 4 muestra las series de tiempo normalizadas para los primeros modos de los modelos del ACC. Para la predicción de la precipitación de MJJ (ASO), se usó como predictor el campo de la TSM de FMA(MJJ). 


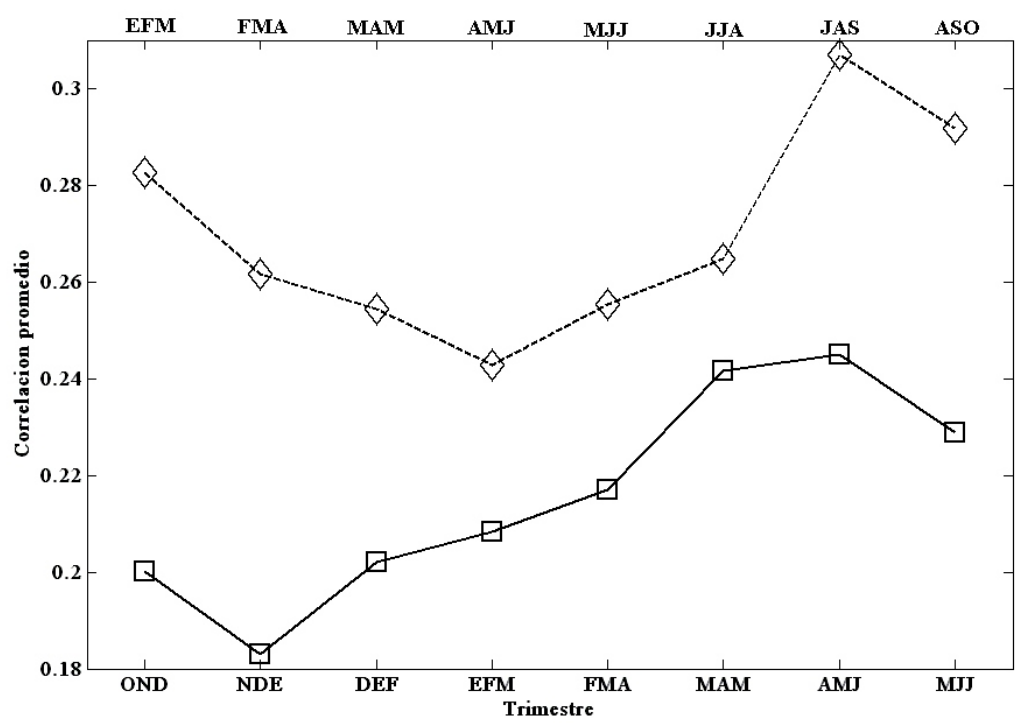

Figura 3. Habilidad estadística predictiva promedio del campo de precipitación en Centroamérica para la predicción de MJJ (linea sólida con cuadros) y para ASO (linea a trazos con rombos). Para la predicción de la precipitación de MJJ, se consideraron los trimestres previos de TSM desde OND hasta AMJ, junto con el trimestre simultáneo de MJJ (eje horizontal inferior), mientras que para la predicción de la precipitación de ASO, se consideraron los trimestres previos de TSM desde EFM hasta JAS, junto con el trimestre simultáneo de ASO (eje horizontal superior).

Las correlaciones entre los modos son de 0.88 (Figura 4a) y 0.87 (Figura 4b), respectivamente, de las cuales sólo la segunda es significativa al $95 \%$, luego de corregir sus grados de libertad debido a la autocorrelación (Davis, 1976). Todas las series mostradas en la Figura 4 mostraron una significancia estadística en su pendiente superior al $99 \%$. Por lo que, en general, este modo se considera que capturó y separó la tendencia lineal presente en el análisis para ambos campos. Esta tendencia, que cruzó el cero en ambos casos alrededor de la década de los 1970, similar a la observada en la oscilación decenal del Pacífico (Mantua et al., 1997), no fue eliminada del análisis por considerarse una fuente importante de variación temporal (Gershunov \& Cayan, 2003; Alfaro et al., 2006). La Figura 5 muestra los segundos modos más correlacionados para la predicción de MJJ. Su correlación fue de 0.72, significativa al 99\% (Davis, 1976). El patrón espacial de este modo, con cada una de las series de tiempo de TSM para FMA, se muestra en la Figura 6. Esta figura, muestra una banda importante de correlaciones positivas que se extiende desde la costa occidental de África hasta $\operatorname{los} 150^{\circ} \mathrm{E}$ y desde $\operatorname{los} 20^{\circ} \mathrm{N}$ hasta el ecuador.
La Figura 7 muestra el patrón espacial de este modo con cada una de las series de tiempo de precipitación en MJJ. Se observa una amplia región de correlaciones positivas desde el norte de Costa Rica hasta Guatemala.

Esto hace suponer que las variaciones positivas (negativas) en la TSM de la franja antes descrita (durante FMA), estarían relacionadas con variaciones positivas (negativas) en la precipitación de MJJ, principalmente por la persistencia de estos patrones oceánicos, en coincidencia con lo observado por Alfaro et al. (1998) y Taylor et al. (2002).

La Tabla 1 muestra la correlación de estos modos con algunos índices de variabilidad climática conocidos para FMA. En esta tabla se observa que el primer modo (Figura 4a, línea sólida), no mostró ninguna correlación significativa con los índices, mientras que el segundo modo (Figura 5, línea sólida), mostró una correlación, significativa al 99\%, con diversos índices del océano Atlántico. Cabe destacar que los modos superiores muestran patrones espaciales más desorganizados, relacionados con fuentes de variación más locales, por lo que los mismos no se muestran. 
a)

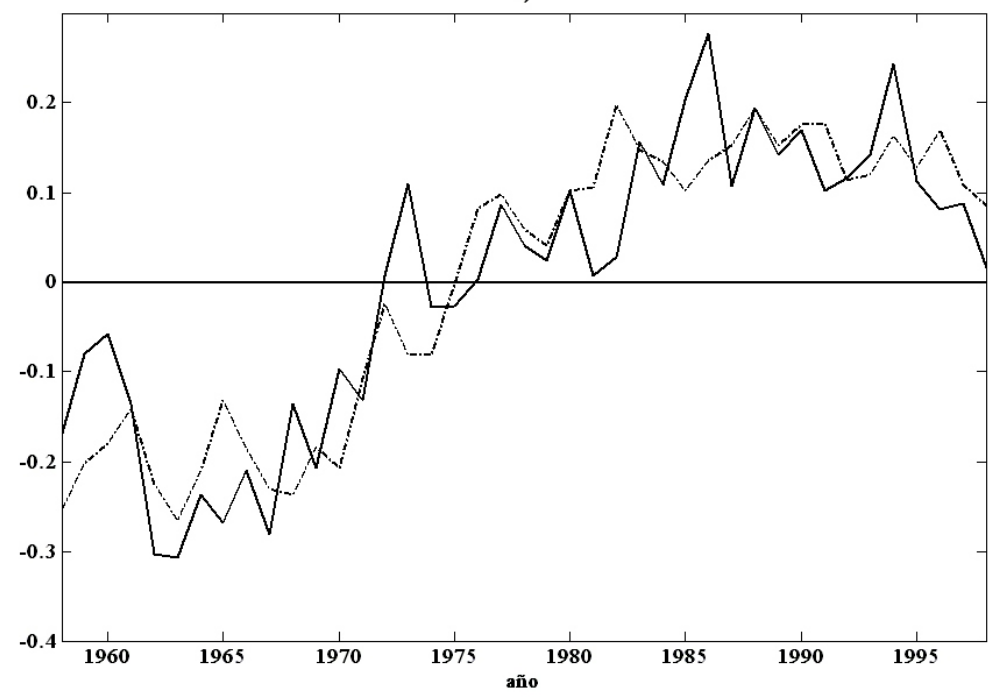

b)

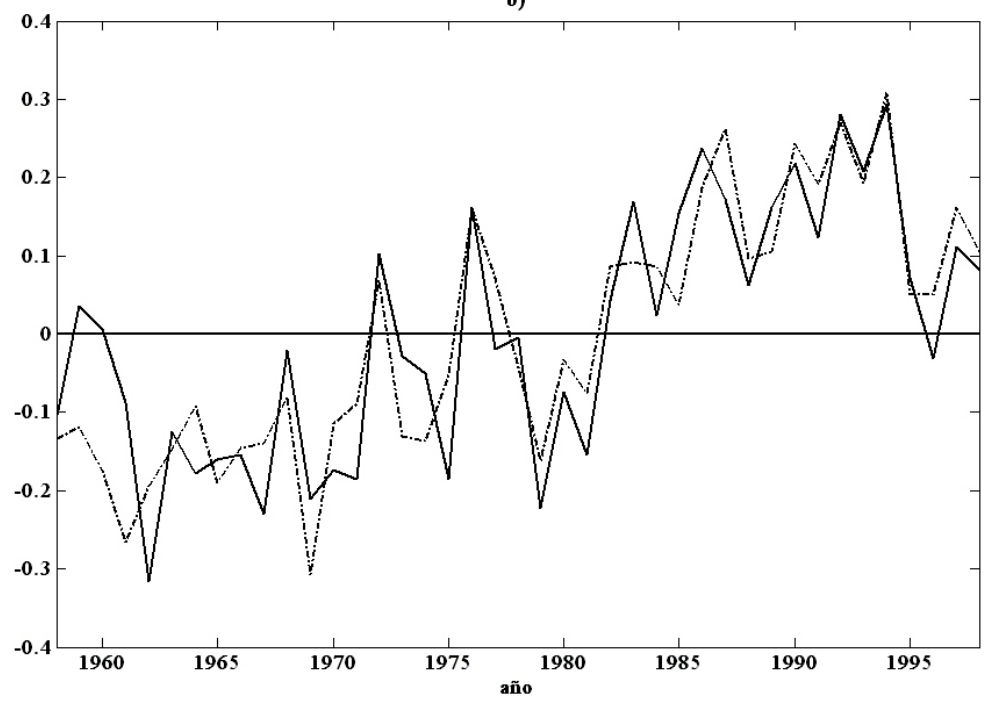

Figura 4. Series de tiempo normalizadas para los primeros modos del ACC: a) MJJ para la precipitación usando la TSM de FMA y b) ASO para la precipitación usando la TSM de MJJ. Las líneas sólidas corresponden a las series anuales de TSM y las punteadas a las de precipitación. Las correlaciones entre los modos son de 0.88 y 0.87 , respectivamente, de las cuales sólo la segunda es significativa al 95\% luego de corregir sus grados de libertad debido a la autocorrelación de las series de acuerdo con Davis (1976). La significancia estadística de la pendiente para todas las series mostradas es superior al $99 \%$. 


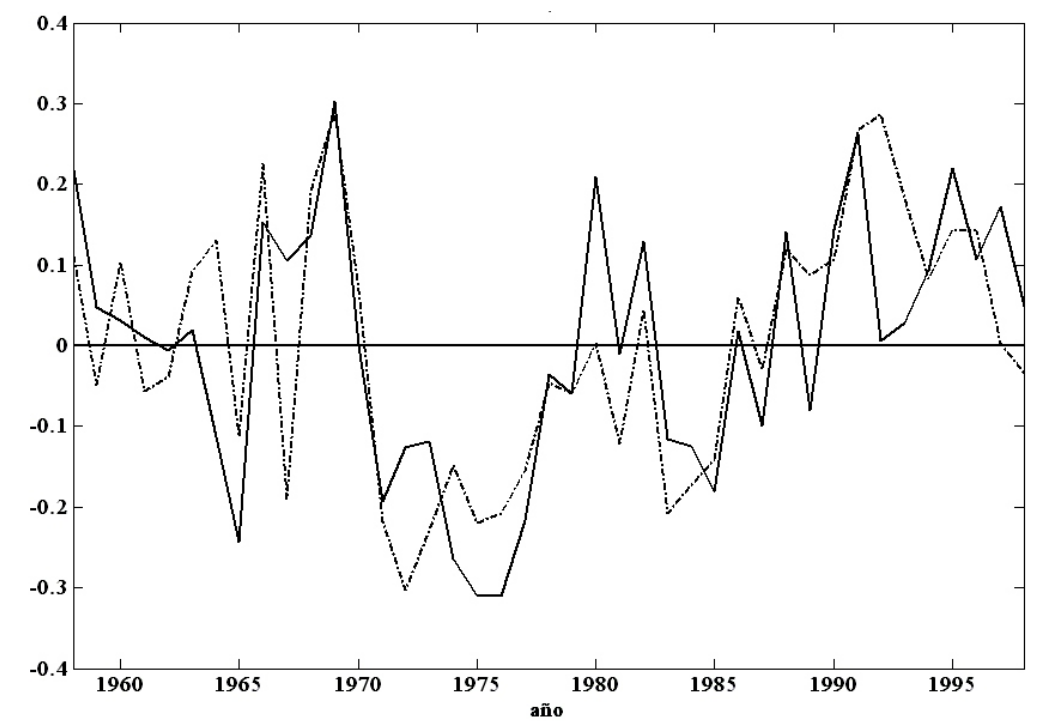

Figura 5. Series de tiempo normalizadas para los segundos modos del ACC, con MJJ para la precipitación usando la TSM de FMA. Las líneas sólidas corresponden a las series anuales de TSM y las punteadas a las de precipitación. La correlación entre los modos es de 0.72, significativa al 99\% de acuerdo con Davis (1976).

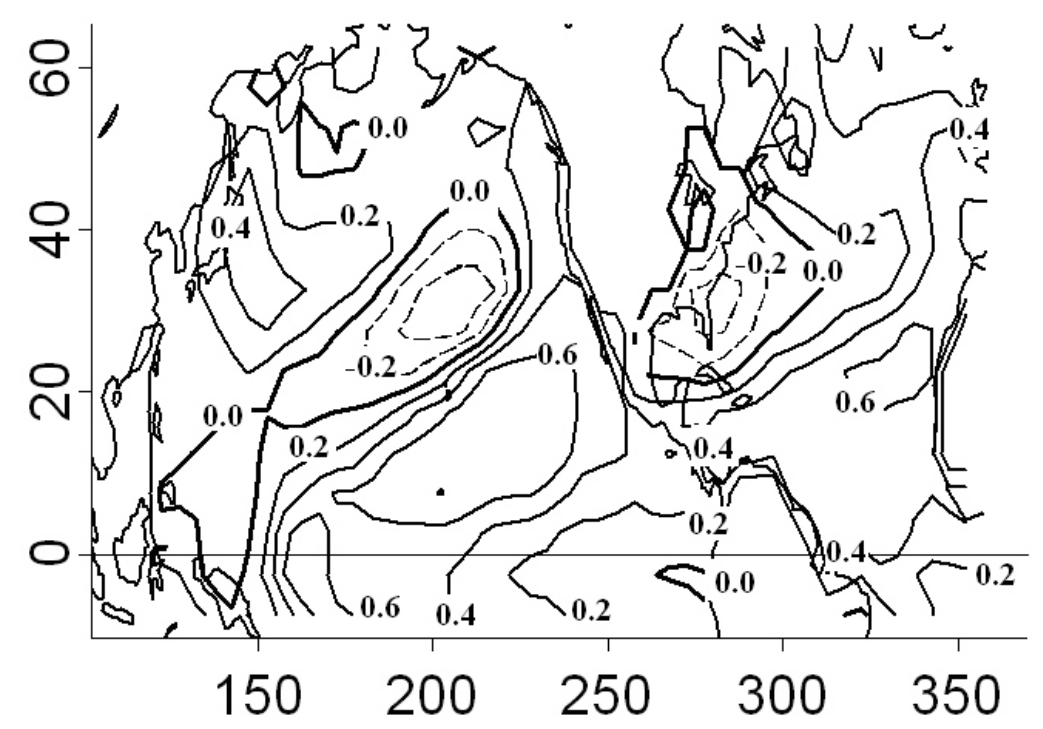

Figura 6. Patrón espacial de correlaciones para el segundo modo de la TSM mostrada en la Figura 5. El contorno más grueso es el de cero correlación. Los contornos se graficaron cada 0.2. Las líneas sólidas son correlaciones positivas y las líneas a trazos son correlaciones negativas. 


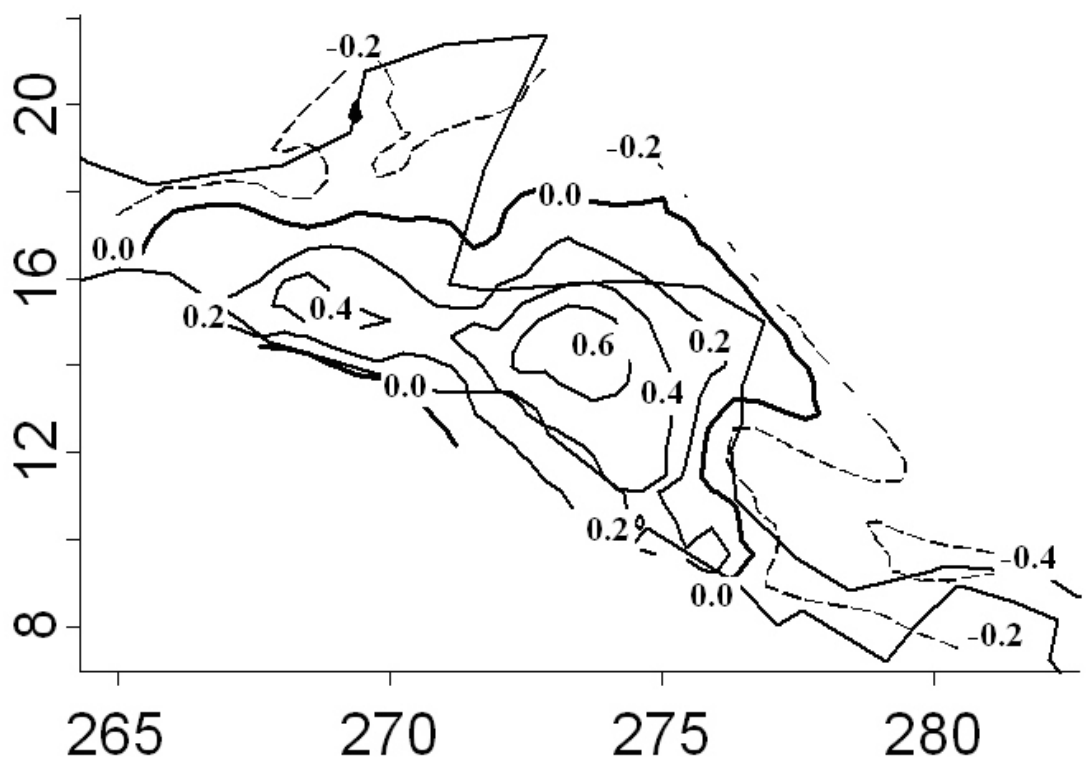

Figura 7. Patrón espacial de correlaciones para el segundo modo de la precipitación mostrada en la Figura 5. El contorno más grueso es el de cero correlación. Los contornos se graficaron cada 0.2. Las líneas sólidas son correlaciones positivas y las líneas a trazos son correlaciones negativas.

Tabla 1. Correlaciones entre los tres primeros modos de TSM y diferentes índices oceánicos. Las correlaciones son para las series de tiempo anuales del trimestre FMA. La operación de suma o resta de los índices oceánicos se realiza sobre las series normalizadas para hacer comparables la variabilidad entre ellas. Se resaltan con negrita los valores máximos de cada columna. Los valores con uno y dos asteriscos son significativos al 95 y $99 \%$ de acuerdo a Davis (1976).

\begin{tabular}{cccc} 
& Modos & TSM & FMA \\
\hline Índices, FMA & 1 & 2 & 3 \\
\hline ODP & -0.5123 & 0.1977 & $\mathbf{0 . 4 7 7 5 *}$ \\
\hline OMA & 0.2823 & $\mathbf{0 . 6 1 9 9 * *}$ & -0.0574 \\
\hline Niño3 & -0.1624 & 0.1773 & 0.1173 \\
\hline ATN & 0.3231 & $0.5521^{* *}$ & $0.4181^{*}$ \\
\hline ODP -OMA & $-\mathbf{0 . 6 9 7 6}$ & -0.3706 & $0.4695^{*}$ \\
\hline Niño3-OMA & -0.4369 & $-0.4349^{*}$ & 0.1717 \\
\hline ODP+Niño3 & -0.3937 & 0.2189 & $0.3471^{*}$
\end{tabular}




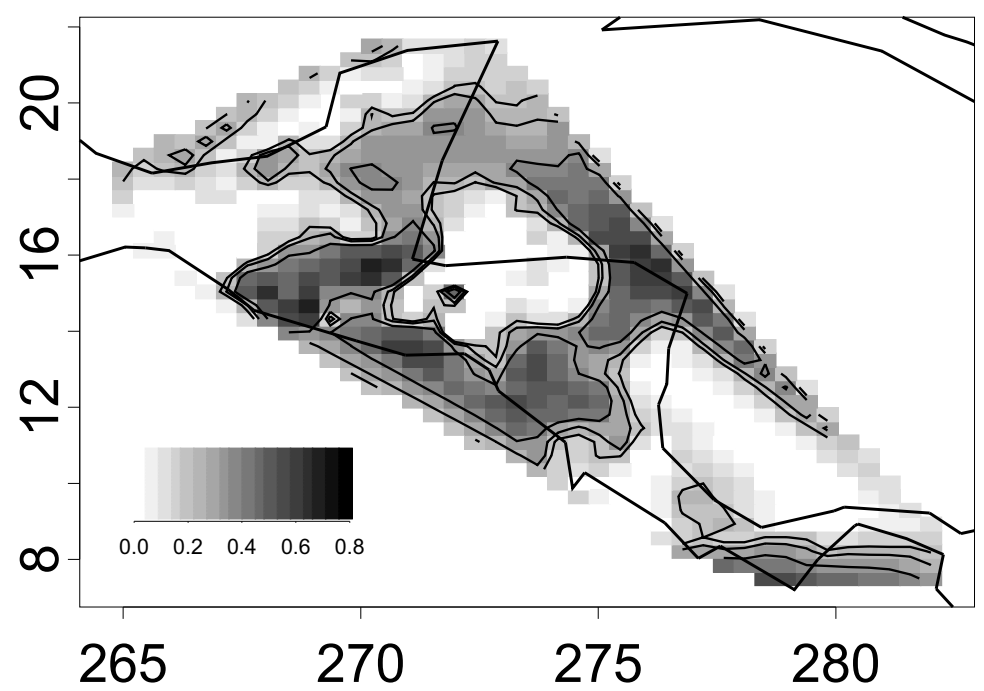

Figura 8. Patrón espacial de la habilidad estadistica predictiva del campo de precipitación en Centroamérica para la predicción del trimestre de MJJ usando la TSM en FMA como predictor. Los valores se calcularon como la correlación entre las series de tiempo observadas y las predichas por el modelo del ACC usando validación cruzada. Los contornos representan los niveles de confianza del 90,95 y $99 \%$ en orden creciente.

La Figura 8 muestra el patrón espacial de la capacidad predictiva del modelo usado para la predicción de MJJ. La región con capacidad predictiva, estadísticamente significativa al $95 \%$, abarca buena parte de Belice, Guatemala, El Salvador, zona central y de la costa Pacífica de Nicaragua, costa nororiental de Honduras y la región cercana a David en Panamá.

Las series de tiempo para el segundo modo más correlacionado en la predicción de ASO se muestran en la Figura 9. Su correlación es de 0.78, significativa a más del 99\% (Davis, 1976). La Figura 10 presenta el patrón espacial de correlaciones entre este modo y las series de tiempo de TSM en MJJ. Este patrón muestra una franja importante de correlaciones positivas que abarca una buena parte del Pacífico ecuatorial y tropical del Este, desde los $170^{\circ} \mathrm{E}$ hasta las costas americanas y desde el sur del dominio utilizado hasta prácticamente $\operatorname{los} 15^{\circ} \mathrm{N}$, aproximadamente. Por otro lado, se encuentra un patrón de correlaciones negativas en prácticamente todo el Atlántico tropical considerado. El patrón espacial de correlaciones entre este modo y las series de tiempo de precipitación en ASO se muestra en la Figura 11. Este patrón muestra un área importante de correlaciones negativas sobre el istmo, exceptuando las costas del Caribe de Honduras, Costa Rica y Panamá en la región de Bocas de Toro, que muestran correlaciones positivas. Este patrón bipolar en el campo de TSM relacionado con variaciones en la precipitación de Centroamérica fue también identificado por Enfield \& Alfaro (1999). En este patrón, importantes variaciones positivas (negativas) en la precipitación están relacionadas con variaciones negativas y positivas (positivas $\mathrm{y}$ negativas) en el Pacífico ecuatorial del Este y en el Atlántico tropical, respectivamente, lo que estaría relacionado con una disminución (un aumento) del flujo alisio del noreste sobre el istmo, lo que actuaría a favor (en contra) de la formación de sistemas mesoescalares, principalmente sobre la vertiente del Pacífico.

El resultado anterior concuerda también con las correlaciones presentadas en la Tabla 2, que muestra la correlación de estos modos con algunos índices de variabilidad climática conocidos para MJJ. Nótese que la mejor correlación encontrada para el segundo modo (Figura 9, línea sólida) fue con la diferencia normalizada de los índices del Niño 3 y la oscilación decenal del Atlántico, significativa al $99 \%$. 


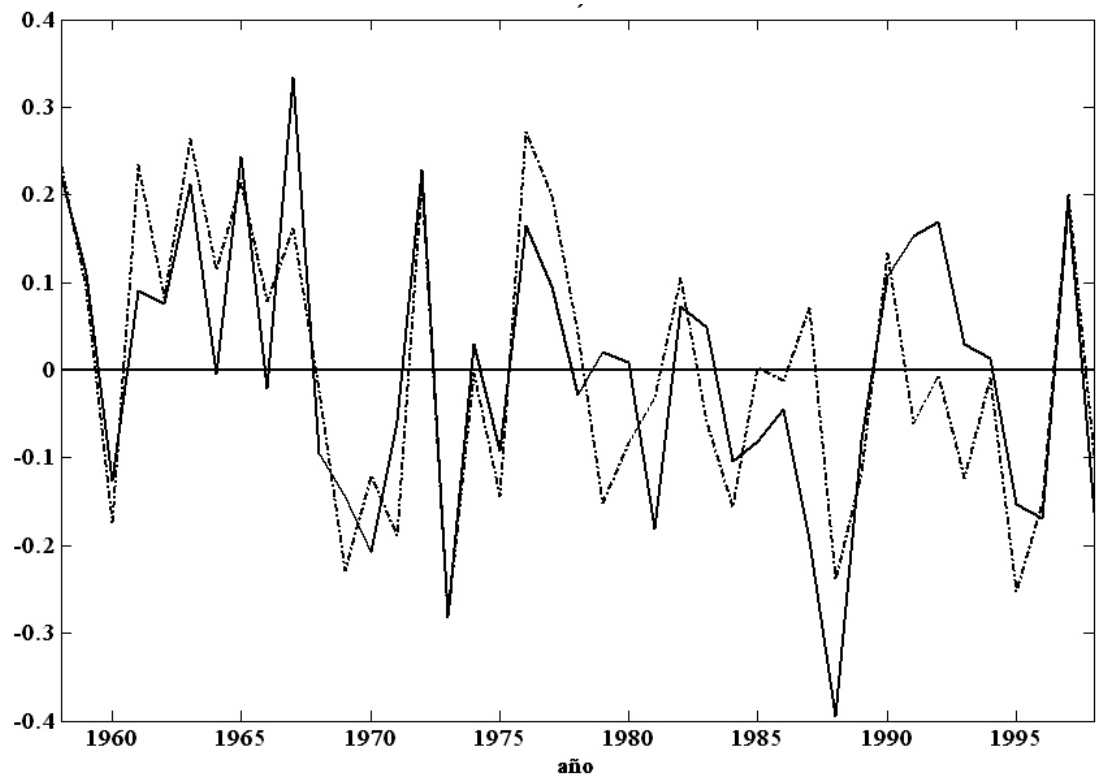

Figura 9. Series de tiempo normalizadas para los segundos modos del ACC, con ASO para la precipitación usando la TSM de MJJ. Las líneas sólidas corresponden a las series anuales de TSM y las punteadas a las de precipitación. La correlación entre los modos es de 0.78 , significativa al 99\% de acuerdo a Davis (1976).

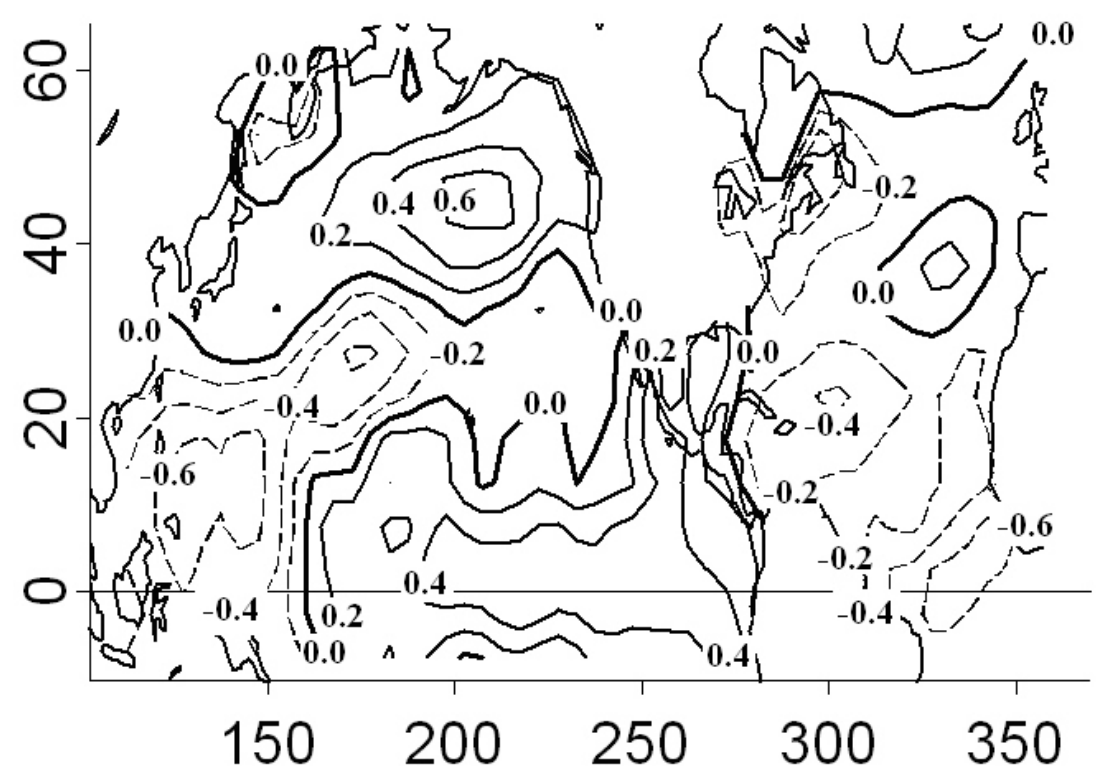

Figura 10. Patrón espacial de correlaciones para el segundo modo de la TSM mostrada en la Figura 9. El contorno más grueso es el de cero correlación. Los contornos se graficaron cada 0.2. Las líneas sólidas son correlaciones positivas y las líneas a trazos son correlaciones negativas. 


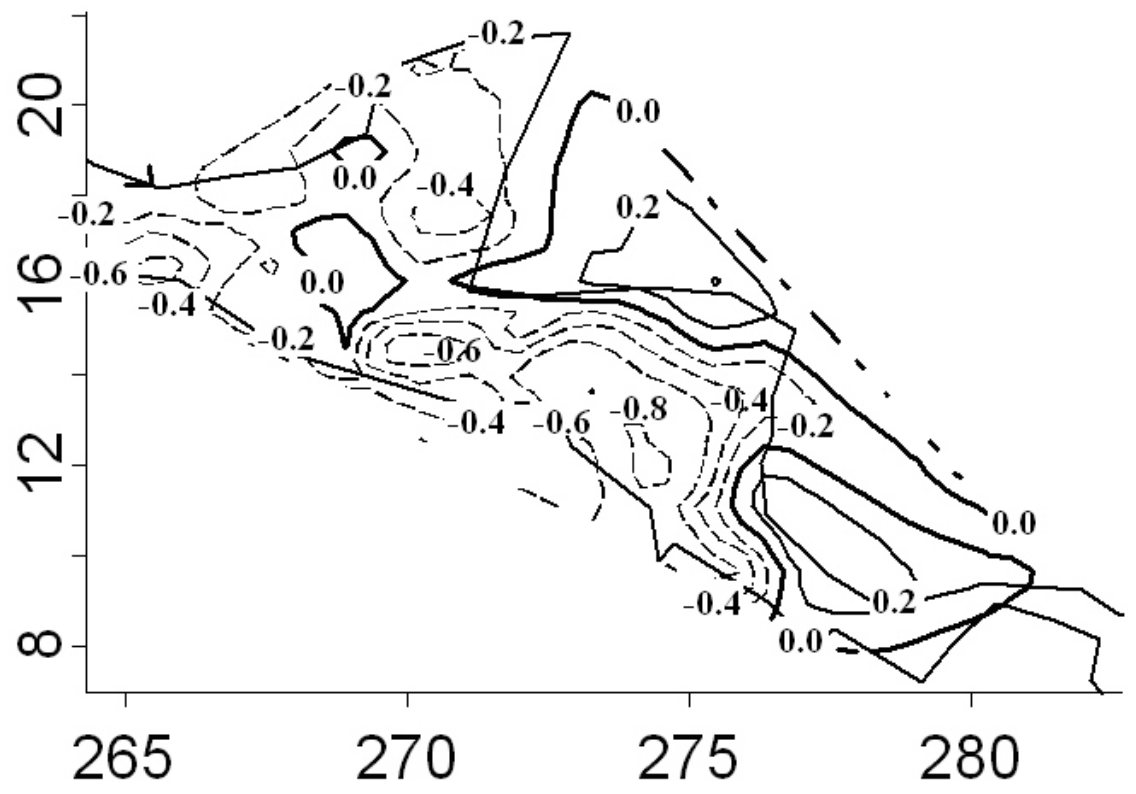

Figura 11. Patrón espacial de correlaciones para el segundo modo de la precipitación mostrada en la Figura 9. El contorno más grueso es el de cero correlación. Los contornos se graficaron cada 0.2. Las líneas sólidas son correlaciones positivas y las líneas a trazos son correlaciones negativas.

Tabla 2. Correlaciones entre los tres primeros modos de TSMy diferentes índices oceánicos para las correlaciones entre las series de tiempo anuales del trimestre MJJ.

\begin{tabular}{cccc} 
& Modos & TSM & MJJ \\
\hline Índices, MJJ & 1 & 2 & 3 \\
\hline ODP & $\mathbf{0 . 5 3 6 9}$ & 0.1935 & 0.249 \\
\hline OMA & 0.0256 & 0.3316 & $\mathbf{0 . 6 4 3 1 *}$ \\
\hline Niño3 & $0.3304^{*}$ & $-0.5404^{* *}$ & -0.0033 \\
\hline ATN & -0.1783 & 0.2257 & $0.4527^{*}$ \\
\hline ODP -OMA & 0.4724 & -0.1276 & -0.3641 \\
\hline Niño3-OMA & 0.2215 & $-\mathbf{0 . 6 3 3 6 * *}$ & $-0.4697^{*}$ \\
\hline ODP+Niño3 & 0.528 & -0.2111 & 0.1496
\end{tabular}




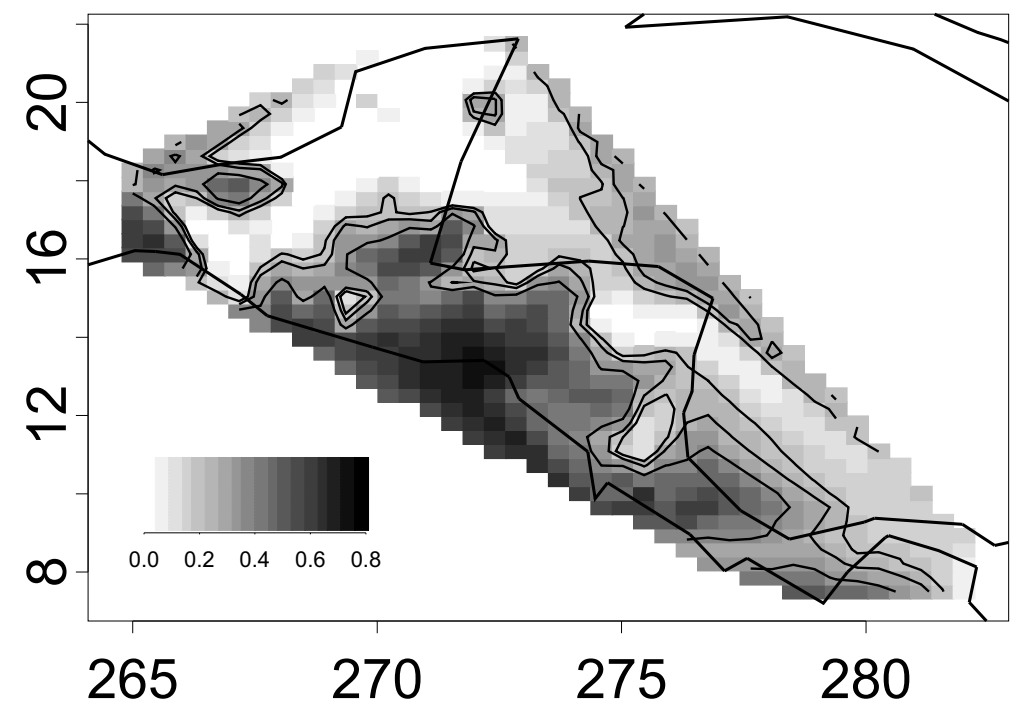

Figura 12. Patrón espacial de la habilidad estadística predictiva del campo de precipitación en Centroamérica para la predicción del trimestre de ASO usando la TSM en MJJ como predictor. Los valores se calcularon como la correlación entre las series de tiempo observadas y las predichas por el modelo del ACC usando validación cruzada. Los contornos representan los niveles de confianza del 90, 95 y 99\% en orden creciente.

Por último, la Figura 12 muestra el patrón la habilidad predictiva del modelo para la precipitación de ASO. Las regiones con correlaciones significativas mayores al $95 \%$ comprenden el sur de Belice, la mayoría del área de Guatemala, El Salvador, las regiones centrales y del Pacífico de Honduras y Nicaragua, el territorio de Costa Rica y la zona más oriental de Panamá.

\section{Conclusiones}

Se observó que la capacidad predictiva de MJJ está asociada con la variabilidad de las TSM en el Atlántico (Tabla 1). Para esta primera parte de la estación lluviosa, se notó que anomalías positivas (negativas) de las TSM en el Atlántico tropical están asociadas con anomalías positivas (negativas) de precipitación sobre un área amplia localizada al norte del istmo (Figura 5). Resultados similares fueron obtenidos por Alfaro et al. (1998), Alfaro \& Cid (1999a), Taylor et al. (2002), Spence et al. (2004) y Ashby et al. (2005), quienes encontraron que el inicio y la primera parte de la estación lluviosa en Centroamérica y el Caribe están influenciadas principalmente por la variabilidad del Atlántico tropical.

Por otra parte, la capacidad predictiva de ASO estuvo influenciada más fuertemente por una especie de dipolo a través del istmo de las TSM, en concordancia con estudios climáticos previos en Mesoamérica tales como los efectuados por Enfield \& Alfaro (1999), Spence et al. (2004) y Ashby et al. (2005) (Tabla 2 y Figura 7). Para esta segunda parte de la estación lluviosa, signos opuestos de las anomalías en los océanos Atlántico y Pacífico, que circundan Centroamérica, estuvieron asociados con una notoria respuesta del campo de precipitación en donde anomalías positivas y negativas (negativas y positivas) de las TSM en los océanos Pacífico y Atlántico tropical, respectivamente, tienden a estar asociadas con lóbulos de anomalías negativas (positivas) de precipitación, principalmente en la vertiente del Pacífico en Centroamérica.

Cabe destacar que tanto para MJJ como para ASO, los principales modos de variación estuvieron asociados a tendencias lineales, relacionadas posiblemente con el cambio de fase que tuvo la oscilación decenal del Pacífico a mediados de la 
década de los 1970 (Gershunov \& Cayan, 2003); sin embargo, no se encontró evidencia estadística significativa para soportar esta afirmación.

Por último, se realizó la validación cruzada de los resultados del modelo para ambas estaciones climáticas, mostrando una habilidad estadísticamente significativa sobre un área importante de la región en estudio, aunque siendo ligeramente mayor el promedio a la asociada con la predicción de ASO.

\section{Agradecimientos}

Este trabajo se realizó gracias al apoyo de los proyectos VI-UCR 805-A2-156, 805-A7-002, 805-98-506, 808-A6-053 y 808-A7-401.

\section{Referencias bibliográficas}

Alfaro, E. (2000). Eventos cálidos y fríos en el Atlántico tropical norte. Atmósfera 13 (2), 109-119.

http://www.ejournal.unam.mx/atmosfera/ Vol13-2/ATM13204.pdf

Alfaro, E. J. (2002). Some characteristics of the annual precipitation cycle in Central America and their relationships with its surrounding tropical oceans. Tópicos Meteorológicos y Oceanográficos 9(2), 88-103.

http://www.imn.ac.cr/publicaciones/revista/ ALFARO\%20FINAL\%2020041202.pdf

Alfaro, E., Cid, L., \& Enfield, D. (1998). Relaciones entre el inicio y el término de la estación lluviosa en Centroamérica y los océanos Pacífico y Atlántico tropical. Investigaciones Marinas 26, 59-69.

http://www.scielo.cl/pdf/imar/v26/art06.pdf

Alfaro, E.J., \& Cid, L. (1999a). Análisis de las anomalías en el inicio y el término de la estación lluviosa en Centroamérica y su relación con los océanos Pacífico y Atlántico tropical. Tópicos Meteorológicos y Oceanográficos 6(1), 1-13. http://www.imn.ac.cr/publicaciones/revista/ Alfaro0799.pdf
Alfaro, E. J., \& Cid, L. (1999b). Ajuste de un modelo VARMA para los campos de anomalías de precipitación en Centroamérica y los índices de los océanos Pacífico y Atlántico tropical. Atmósfera 12 (4), 205-222.

http://www.ejournal.unam.mx/atmosfera/Vol124/ATM12403.pdf

Alfaro, E. J., \& Soley, F.J. (2001). Ajuste de un modelo VAR como predictor de los campos de anomalías de precipitación en Centroamérica. Revista de Matemática: Teoría y Aplicaciones $8(1), 77-94$.

http://revista.emate.ucr.ac.cr/Numerosanteriores/ 2001/Vol8n1/Alfaro.pdf

Alfaro, E. J., Cayan, D., \& Gershunov, A. (2006). Prediction of summer maximum and minimum temperature over the central and western United States: the roles of soil moisture and sea surface temperature. Journal of Climate 19 (8), 14071421.

http://ams.allenpress.com/perlserv/?request=getarchive \&issn $=1520-0442 \& \mathrm{ct}=1$

Amador, J.A. (1998). A climatic feature of tropical Americas: the trade wind easterly jet. Tópicos Meteorológicos y Oceanográficos 5 (2), 91-102.

Ashby, S., Taylor, M. A., \& Chen, A. A. (2005). Statistical models for predicting rainfall in the Caribbean. Theoretical and Applied Climatology $82(1 / 2), 65-80$.

Barry, R. G., \& Carleton, A. M. (2001). Synoptic and dynamic climatology. New York: Routledge.

Davis, R.E. (1976). Predictability of sea surface temperature and sea level pressure anomalies over the North Pacific ocean. Journal of Physical Oceanography 6(3), 249-266.

$\mathrm{http}: / /$ ams.allenpress.com/perlserv/?SESSID $=16$ a2f8648dfd709bf0ca15a6313b00d3\&request $=$ get-archive\&issn=1520-0485 
Enfield, D. B., \& Alfaro, E. J. (1999). The dependence of Caribbean rainfall on the interaction of the tropical Atlantic and Pacific oceans. Journal of Climate 12 (7), 2093-2103. http://ams.allenpress.com/perlserv/?request=getarchive \&issn $=1520-0442 \& \mathrm{ct}=1$

Enfield, D.B., Mestas-Nuñez, A.M., \& Trimble, P.J. (2001). The Atlantic multidecadal oscillation and its relation to rainfall and river flows in the continental U.S. Geophysical Research Letters 28 (10), 2077-2080.

http://www.aoml.noaa.gov/phod/docs/enfield/ enfield_etal2001.pdf

Gershunov, A., \& Cayan, D.R. (2003). Heavy daily precipitation frequency over the contiguous United States: sources of climate variability and seasonal predictability. Journal of Climate 16(16), 2752-2765.

http://ams.allenpress.com/perlserv/?request=getarchive \&issn $=1520-0442 \& \mathrm{ct}=1$

Giannini, A., Kushnir, Y. \& Cane, M.A. (2000). Interannual variability of Caribbean rainfall, ENSO, and the Atlantic ocean. Journal of Climate 13 (2), 297-311.

http://ams.allenpress.com/perlserv/?request=getarchive \&issn $=1520-0442 \& \mathrm{ct}=1$

Giannini, A., Cane, M.A., \& Kushnir, Y. (2001). Interdecadal changes in the ENSO teleconnection to the Caribbean region and the North Atlantic oscillation. Journal of Climate 14 (13), 28672879.

http://ams.allenpress.com/perlserv/?request=getarchive \&issn $=1520-0442 \& \mathrm{ct}=1$

Kaplan, A., Cane, M. A., Kushnir, Y., Clement, A.C., Blumenthal, M. B., \& Rajagopalan, B. (1998). Analysis of global sea surface temperatures 1856-1991. Journal of Geophysical Research-Oceans 103 (C9), 18567-18590.

Magaña, V., Amador, J., \& Medina, S. (1999). The midsummer drougth over Mexico and Central America. Journal of Climate 12 (6), 1577-1588. $\mathrm{http}: / /$ ams.allenpress.com/perlserv/?request $=$ getarchive \&issn $=1520-0442 \& \mathrm{ct}=1$
Mantua, N. J., Hare, S.R., Zhang, Y., Wallace, J.M., \& Francis, R.C. (1997). A Pacific interdecadal climate oscillation with impacts on salmon production. Bulletin of the American Meteorological Society 78 (6), 1069-1079.

$\mathrm{http}: / /$ ams.allenpress.com/perlserv/?SESSID $=16$ a2f8648dfd709bf0ca15a6313b00d3\&request $=$ get-archive \&issn=1520-0477

Poveda, G., \& Mesa, O.J. (1999). La corriente de chorro superficial del oeste [del Chocó] y otras dos corrientes de chorro en Colombia: climatología y variabilidad durante las fases del ENSO. Revista de la Academia Colombiana de Ciencias Exactas, Físicas y Naturales 23 (89), 517-528.

Poveda, G., Waylen, P. R., \& Pulwarty, R. S. (2006). Annual and inter-annual variability of the present climate in northern South America and southern Mesoamerica. Palaeogeography, Palaeoclimatology, and Palaeoecology 234 (1), 3-27.

Reynolds, R. W., \& Smith, T. (1994). Improved global sea surface temperature analyses using optimum interpolation. Journal of Climate 7 (6), 929-948.

http://ams.allenpress.com/perlserv/?request=getarchive\&issn $=1520-0442 \& c t=1$

Spence, J.M., Taylor, M.A., \& Chen, A.A. (2004). The effect of concurrent sea-surface temperature anomalies in the tropical Pacific and Atlantic on Caribbean rainfall. International Journal of Climatology 24(12), 1531-1541.

Taylor, M. A., Enfield, D. B., \& Chen, A. A. (2002). The influence of the tropical Atlantic versus the tropical Pacific on Caribbean rainfall. Journal of Geophysical Research-Oceans 107(C9), 10.1-10.14.

Taylor, M. A., \& Alfaro, E. J. (2005). Climate of Central America and the Caribbean. In: J. E. Oliver (editor) Encyclopedia of World Climatology. Netherlands: Springer, p. 183-188.

Wang, C.(2006). An overlooked feature of tropical climate: inter-Pacific-Atlantic variability. Geophysical Research Letters 33 (12), L12702.1L12702.5. 
Wang, C., Enfield, D.B., Lee, S.-K., \& Landsea, C.W. (2006). Influences of the Atlantic warm pool on western hemisphere summer rainfall and Atlantic hurricanes. Journal of Climate 19 (12), 3011-3028.

http://ams.allenpress.com/perlserv/?request=getarchive \&issn $=1520-0442 \& c t=1$

Wang, C. (2007). Variability of the Caribbean lowlevel jet and its relations to climate. Climate Dynamics 29(4), 411-422.

http://www.aoml.noaa.gov/phod/docs/wang_e06 59.pdf

Waylen, P. R., Quesada, M. E., \& Caviedes, C. N. (1994). The effects of El Niño-Southern oscillation on precipitation in San Jose, Costa Rica. International Journal of Climatology 14 (5), 559-568.

Waylen, P. R., Caviedes, C. N., \& Quesada, M. E. (1996a). Interannual variability of monthly precipitation in Costa Rica. Journal of Climate 9(10), 2606-2613.

http://ams.allenpress.com/perlserv/?request=getarchive \&issn $=1520-0442 \& c t=1$

Waylen, P. R., Quesada, M. E., \& Caviedes, C. N. (1996b). Temporal and spatial variability of annual precipitation in Costa Rica and the southern oscillation. International Journal of Climatology 16(2), 173-193.

Waylen, P.R., \& Quesada, M. (2001). The effect of Atlantic and Pacific surface temperatures on the mid-summer drought of Costa Rica. Cuadernos de Investigación Geográfica No. 27, 193-205.

http://dialnet.unirioja.es/servlet/fichero_articulo? codigo $=258504 \&$ orden $=74448$

Wilks, D. S. (1995). Statistical Methods in the Atmospheric Sciences. Academic Press. 